\title{
Development of the Approximating Functions Method for Problems in a Planar Waveguide with Constant Polarization
}

\author{
Denis Zolotariov', Alexander Nerukh ${ }^{2}$ \\ ${ }^{1}$ Ph.D., Kharkov, Ukraine, https://orcid.org/0000-0003-4907-7810 \\ ${ }^{2}$ Prof., Kharkov National University of Radio Electronics, Kharkov, Ukraine, https://orcid.org/0000-0003-0934-2237
}

\begin{tabular}{ll}
\hline ARTICLE INFO & ABSTRACT \\
\hline $\begin{array}{l}\text { Published Online } \\
\text { 01 December 2021 }\end{array}$ & $\begin{array}{l}\text { Article introduces an extension of the approximating functions method, a particular case of the } \\
\text { finite element method (FEM) with interpolating functions in the form of Lagrange polynomials of a } \\
\text { special form, to solve electrodynamics problems in a planar waveguide with constant polarization in } \\
\text { the spatial-temporal domain using the Volterra integral equation method. The main goal of the } \\
\text { article is to expand the area of applicability of this method to three-dimensional problems in a }\end{array}$ \\
Corresponding Author: & $\begin{array}{l}\text { planar waveguide with constant polarization, as well as to obtain general interpolation expressions } \\
\text { in analytical form, which will be used to construct a system of nonlinear equations for solving } \\
\text { Denis Zolotariov, }\end{array}$ \\
\hline
\end{tabular}

KEYWORDS: Approximating functions method, Volterra integral equation method, Planar waveguide

\section{INTRODUCTION}

The Volterra integral equation method is an approach based on integral equations equivalent to the Maxwell's equations [1-2] to solve electrodynamics problems in 1-3 dimensional space and time domain. Its key features are natural description of non-stationary and nonlinear features, unified definition of the inner and outer problems, and inclusion of initial and boundary conditions in the same equations - the equations form is Volterra integral equation of the second kind and it is the same for various media and does not depend on the initial signal expression. These advantages of the Volterra integral equation method significantly simplify the problem statement and its solution process, based on the universal modeling algorithms for a wide range of electrodynamics problems [3-4]. The most qualitative and promising solution is based on the analytical-numerical method of approximating functions.

The approximating functions method is a particular case of the finite element method [5-7] with partitioning the problem definition domain by cells and approximation of the desired function by Lagrange polynomials of the second order in each of them. Calculation of the Volterra equation with such approximation at the mesh points reduces the solving of the problem to finding a solution to the corresponding system of nonlinear algebraic equations. The system is solved by Newton's method or any other method suitable for these purposes.

The approximating functions method was originally proposed for general one-dimensional case in [8-9], and was extended for solving electrodynamics problems by Volterra integral equation method in two-dimensional (1D-spatial and time) region in [10-11]. The article [12] proposed an approach to construct fault-tolerant computing scheme to improve the performance and reliability of the latter, which was later used to build a microservice calculation node in a microservice application in [13-14].

In work [15], the approximating functions method was extended for modeling problems in the three-dimensional space in a planar waveguide with non-magnetic media with losses, which makes it possible to solve a much wider range of problems, including problems with media with nonstationary and non-linear properties.

The present work continues to develop the approximating functions method for the planar waveguides with non-magnetic media with losses for the case of constant polarization inside it.

\section{PROBLEM STATEMENT}

As it was shown in work [15], the equation for the problem with planar waveguide with non-magnetic media with 


\section{"Development of the Approximating Functions Method for Problems in a Planar Waveguide with Constant Polarization"}

losses, where signal propagating along $z$ axis only with no dependency on a transversal $y$ coordinate, according to the Volterra integral equation method has a form:

$\mathbf{E}=\mathbf{E}_{0}+$

$+\frac{1}{4 \pi}\left(\nabla \nabla-\frac{1}{v^{2}} \frac{\partial^{2}}{\partial t^{2}} \hat{I}\right) \int_{0}^{\infty} d t^{\prime} \int_{-\infty}^{\infty} d z^{\prime} \int_{-\infty}^{\infty} d x^{\prime} \frac{2 v}{\sqrt{v^{2} \square t^{2}-R_{\perp}^{2}}} \times$

$\times \theta\left(v^{2} \square t^{2}-R_{\perp}^{2}\right) \chi\left\{\frac{1}{\varepsilon_{0} \varepsilon} \mathbf{P}+v^{2} \mu_{0} \int_{0}^{t^{\prime}} d t^{\prime \prime} \mathbf{j}\right\}$

where $\mathbf{E}_{0}$ is an undisturbed electric field, $\mathbf{P}$ is a polarization of the medium inside the slab, which has different electromagnetic characteristics than the environment outside it, $\mathbf{j}$ is a conductivity current inside the slab,$\varepsilon$ is the permittivity of medium in the environment, $v=c / \sqrt{\varepsilon}$ and the SI system of units is used. The function $\chi$ equals 1 inside the waveguide and 0 outside it, $\nabla \nabla$ is a Hessian matrix, $R_{\perp}^{2}=\left(x-x^{\prime}\right)^{2}+\left(z-z^{\prime}\right)^{2}$ and $\square t=t-t^{\prime}$.

Also, in accordance with [15], the field in waveguide is expanded into its longitudinal and transverse components, presented in Equation (2) and (3) below:

$E_{z}=E_{0 z}+$

$+\frac{1}{4 \pi}\left(\partial_{z z}^{2}-\frac{1}{v^{2}} \frac{\partial^{2}}{\partial t^{2}}\right) \int_{0}^{\infty} d t^{\prime} \int_{-\infty}^{\infty} d z^{\prime} \int_{-\infty}^{\infty} d x^{\prime} \frac{2 v}{\sqrt{v^{2} \square t^{2}-R_{\perp}^{2}}} \times$

$\times \theta\left(v^{2} \square t^{2}-R_{\perp}^{2}\right) \chi\left\{\frac{1}{\varepsilon_{0} \varepsilon} P_{z}+v^{2} \mu_{0} \int_{0}^{t^{\prime}} d t^{\prime \prime} j_{z}\right\}+$

$+\frac{1}{4 \pi}\left(\partial_{z x}^{2}\right) \int_{0}^{\infty} d t^{\prime} \int_{-\infty}^{\infty} d z^{\prime} \int_{-\infty}^{\infty} d x^{\prime} \frac{2 v}{\sqrt{v^{2} \square t^{2}-R_{\perp}^{2}}} \times$

$\times \theta\left(v^{2} \square t^{2}-R_{\perp}^{2}\right) \chi\left\{\frac{1}{\varepsilon_{0} \varepsilon} P_{x}+v^{2} \mu_{0} \int_{0}^{t^{\prime}} d t^{\prime \prime} j_{x}\right\}$

$E_{x}=E_{0 x}+$

$+\frac{1}{4 \pi}\left(\partial_{x x}^{2}-\frac{1}{v^{2}} \frac{\partial^{2}}{\partial t^{2}}\right) \int_{0}^{\infty} d t^{\prime} \int_{-\infty}^{\infty} d z^{\prime} \int_{-\infty}^{\infty} d x^{\prime} \frac{2 v}{\sqrt{v^{2} \square t^{2}-R_{\perp}^{2}}} \times$

$\times \theta\left(v^{2} \square t^{2}-R_{\perp}^{2}\right) \chi\left\{\frac{1}{\varepsilon_{0} \varepsilon} P_{x}+v^{2} \mu_{0} \int_{0}^{t^{\prime}} d t " j_{x}\right\}+$

$+\frac{1}{4 \pi}\left(\partial_{x z}^{2}\right) \int_{0}^{\infty} d t^{\prime} \int_{-\infty}^{\infty} d z^{\prime} \int_{-\infty}^{\infty} d x^{\prime} \frac{2 v}{\sqrt{v^{2} \square t^{2}-R_{\perp}^{2}}} \times$

$\times \theta\left(v^{2} \square t^{2}-R_{\perp}^{2}\right) \chi\left\{\frac{1}{\varepsilon_{0} \varepsilon} P_{z}+v^{2} \mu_{0} \int_{0}^{t^{\prime}} d t^{\prime \prime} j_{z}\right\}$

These equations describe the electromagnetic field evolution in time interval $t \in[0, \infty)$. If the observation point $(z, x)$ belongs to the slab $x \in\left[x_{\min }, x_{\max }\right]$, then they formulate the problem for the internal field. Outside it, the expressions are quadrature formulas for calculating the fields in the cladding, taking into account the field in the waveguide.
The integrating area for internal problem is specified by two inequalities:

$v^{2}\left(t-t^{\prime}\right)^{2}-\left(x-x^{\prime}\right)^{2}-\left(z-z^{\prime}\right)^{2}>0$ and $\chi=1$,

and for some point $(t, x, z)$ is presented on Fig. 1 as a semitransparent blue cone with a base that is described by a circle $\left(x-x^{\prime}\right)^{2}+\left(z-z^{\prime}\right)^{2}=v^{2}\left(t-t^{\prime}\right)^{2}$. It is clearly seen that the integration region is cut off by the waveguide wall $x=x_{\max }$ due to the influence of equality $\chi=1$.

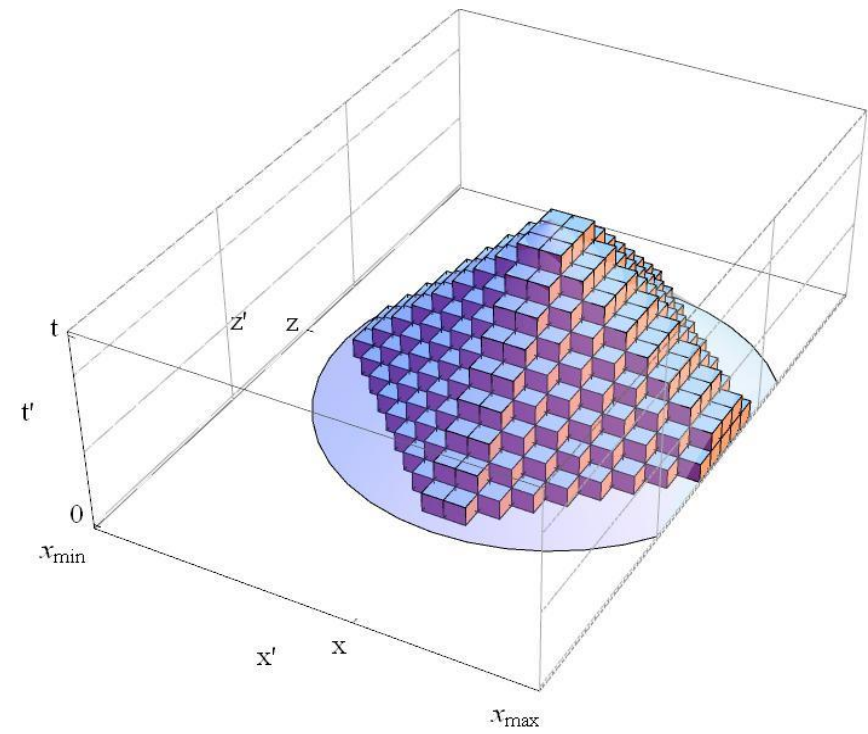

Fig.1. Cone of integration in slab for some point $(t, x, z)$

\section{APPLICATION OF THE APPROXIMATING FUNCTIONS METHOD}

As is known from [10-11], for application of the approximating functions method, the domain of the problem $D$ should be divided into cells-cuboids $D_{i, j, k}$ :

$D=\bigcup D_{i, j, k}$,

$D_{i, j, k}=\left\{\begin{array}{l}i h \leq t<(i+1) h, \\ j h \leq x<(j+1) h, \\ k h \leq z<(k+1) h\end{array}\right\}$,

$i=\overline{0, \mathrm{n}-1}, \quad j=\overline{0, \mathrm{~m}-1}, \quad k=\overline{0, \mathrm{p}-1}$,

where $h$ is a spacing of the mesh. Such an approximation of the region $D$ by cuboids $D_{i, j, k}$ is shown in Fig. 1 in the form of a set of cubes, located on top of each other, which fill the cone, but do not go beyond it.

The solutions to Equation (2) and (3) are constructed approximately as a sum of piecewise-smooth functions $\hat{E}_{i, j, k}(t, x, z)$ each of which is determined in the corresponding mesh cell $D_{i, j, k}$, which is presented on Fig. 2.

To successfully solve Equation (2) and (3) by approximating functions method, the integrals in them must be calculated as analytical expressions. Eliminating the 


\section{"Development of the Approximating Functions Method for Problems in a Planar Waveguide with Constant Polarization"}

constants, we can say that only an expression with polarization $P$ can affect this.

Let's consider this expression separately in one cell of the mesh, which will have the following form:

$$
I=\int_{t_{i}}^{t_{i}+h} d t^{\prime} \int_{x_{j}}^{x_{j}+h} d x^{\prime} \int_{z_{k}}^{z_{k}+h} d z^{\prime} \frac{P\left(t^{\prime}, x^{\prime}, z^{\prime}\right)}{\sqrt{v^{2}\left(t-t^{\prime}\right)^{2}-\left(x-x^{\prime}\right)^{2}-\left(z-z^{\prime}\right)^{2}}}
$$

For the convenience of further reasoning, let's introduce a change of variables:

$I=-\frac{1}{v} \int_{T_{i}}^{T_{i h}} d t^{\prime} \int_{X_{j}}^{X_{j h}} d x^{\prime} \int_{Z_{k}}^{Z_{k h}} d z^{\prime} \frac{P\left(t-t^{\prime \prime} / v, x-x^{\prime \prime}, z-z^{\prime \prime}\right)}{\sqrt{t^{\prime 2}-x^{\prime \prime}-z^{\prime 2}}}$,

where $v\left(t-t^{\prime}\right)=t^{\prime \prime}, \quad x^{\prime \prime}=x-x^{\prime}, \quad z^{\prime \prime}=z-z^{\prime}, \quad T_{i}=t-t_{i}$, $T_{i h}=t-t_{i}-h, \quad X_{j}=x-x_{j}, \quad X_{j h}=x-x_{j}-h, \quad Z_{k}=z-z_{k}$, $Z_{k h}=z-z_{k}-h$.

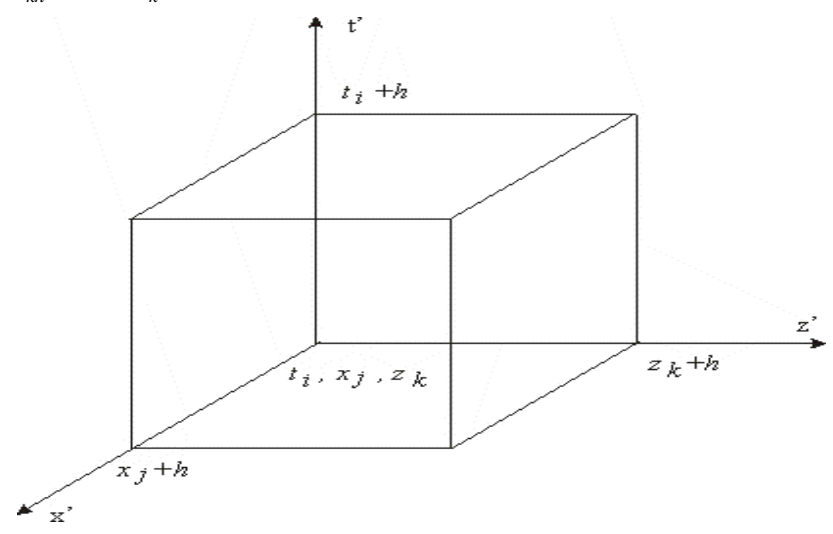

Fig.2. One cell $D_{i, j, k}$ of the mesh

In the general case, the integral in Equation (7) cannot be calculated in the form of an analytical expression, so let's consider only the situation with constant polarization $P\left(t^{\prime}, x^{\prime}, z^{\prime}\right)=$ const , and obtain an integral value for it.

IV. CASE WITH CONSTANT POLARIZATION

To calculate the integral, we will calculate it sequentially from the inside out. Then the innermost integral will have the following analytical representation:

$$
\begin{aligned}
& \int_{Z_{k}}^{Z_{k h}} d z^{\prime \prime} \frac{1}{\sqrt{t^{\prime \prime 2}-x^{\prime 2}-z^{\prime \prime 2}}}= \\
& =\arcsin \frac{Z_{k h}}{\sqrt{t^{\prime \prime 2}-x^{\prime \prime 2}}}-\arcsin \frac{Z_{k}}{\sqrt{t^{\prime 2}-x^{\prime 2}}}= \\
& =\arcsin \frac{Z_{k h} \sqrt{t^{\prime \prime 2}-x^{\prime \prime 2}-Z_{k}^{2}}-Z_{k} \sqrt{t^{\prime \prime 2}-x^{\prime \prime 2}-Z_{k h}^{2}}}{t^{\prime \prime 2}-x^{\prime \prime 2}}
\end{aligned}
$$

where used the identity

$$
\arcsin a-\arcsin b=\arcsin \left(a \sqrt{1-b^{2}}-b \sqrt{1-a^{2}}\right) \text {. }
$$

Since the mesh step $h$ is a very small value, the next expression is applicable:

$\sqrt{t^{\prime \prime 2}-x^{\prime \prime 2}-\left(Z_{k}-h\right)^{2}} \approx$

$\sqrt{t^{\prime \prime 2}-x^{\prime \prime 2}-Z_{k}^{2}}+\frac{Z_{k} h}{\sqrt{t^{\prime 2}-x^{\prime 2}-Z_{k}^{2}}}$ and the final expression for Equation (8) will have the following form:

$$
\int_{Z_{k}}^{Z_{k h}} d z^{\prime \prime} \frac{1}{\sqrt{t^{\prime 2}-x^{\prime \prime 2}-z^{\prime \prime 2}}} \approx-\arcsin \frac{h}{\sqrt{t^{\prime 2}-x^{\prime 2}-Z_{k}^{2}}}
$$

Next, consider the integral over the $x$ coordinate. Integrating by it gives us:

$$
\begin{aligned}
& \int_{X_{j}}^{X_{j h}} d x \arcsin \frac{Z_{k h}}{\sqrt{t^{\prime \prime 2}-x^{\prime 2}}}=X_{j h} \arcsin \frac{Z_{k h}}{\sqrt{t^{\prime 2}-X_{j h}^{2}}}- \\
& -X_{j} \arcsin \frac{Z_{k h}}{\sqrt{t^{\prime \prime 2}-X_{j}^{2}}}+Z_{k h} \arcsin \frac{X_{j h}}{\sqrt{t^{\prime \prime 2}-Z_{k h}^{2}}}- \\
& -Z_{k h} \arcsin \frac{X_{j}}{\sqrt{t^{\prime \prime 2}-Z_{k h}^{2}}}- \\
& -\frac{\left|Z_{k h}\right| t^{\prime \prime}}{Z_{k h}} \arcsin \frac{X_{j h} Z_{k h}}{\sqrt{t^{\prime \prime 2}-Z_{k h}^{2}} \sqrt{t^{\prime \prime 2}-X_{j h}^{2}}}+ \\
& +\frac{\left|Z_{k h}\right| t^{\prime \prime}}{Z_{k h}} \arcsin \frac{X_{j} Z_{k h}}{\sqrt{t^{\prime 2}-Z_{k h}^{2}} \sqrt{t^{\prime \prime 2}-X_{j}^{2}}},
\end{aligned}
$$

where used the formulae from [16]:

$\int \frac{d x}{\left(b^{2}-x^{2}\right) \sqrt{a^{2}-x^{2}}}=\frac{1}{b \sqrt{b^{2}-a^{2}}} \arcsin \frac{x \sqrt{b^{2}-a^{2}}}{a \sqrt{b^{2}-x^{2}}}$,

where $a^{2}<b^{2}$.

Further, the double integral of $x$ and $z$ will have the following analytical representation:

$$
\begin{aligned}
& \int_{X_{j}}^{X_{j h}} d x " \int_{Z_{k}}^{Z_{k h}} d z^{\prime \prime} \frac{1}{\sqrt{t^{\prime 2}-x^{\prime 2}-z^{\prime 2}}}= \\
& X_{j h}\left(\arcsin \frac{Z_{k h}}{\sqrt{t^{\prime 2}-X_{j h}^{2}}}-\arcsin \frac{Z_{k}}{\sqrt{t^{\prime 2}-X_{j h}^{2}}}\right)- \\
& -X_{j}\left(\arcsin \frac{Z_{k h}}{\sqrt{t^{\prime 2}-X_{j}^{2}}}-\arcsin \frac{Z_{k}}{\sqrt{t^{\prime 2}-X_{j}^{2}}}\right)+ \\
& +Z_{k h}\left(\arcsin \frac{X_{j h}}{\sqrt{t^{\prime 2}-Z_{k h}^{2}}}-\arcsin \frac{X_{j}}{\sqrt{t^{\prime 2}-Z_{k h}^{2}}}\right)- \\
& -Z_{k}\left(\arcsin \frac{X_{j h}}{\sqrt{t^{\prime^{2}}-Z_{k}^{2}}}-\arcsin \frac{X_{j}}{\sqrt{t^{\prime^{2}}-Z_{k}^{2}}}\right)- \\
& -\frac{\left|Z_{k h}\right| t^{\prime \prime}}{Z_{k h}}\left(\begin{array}{c}
\arcsin \frac{X_{j h} Z_{k h}}{\sqrt{t^{\prime 2}-Z_{k h}^{2}} \sqrt{t^{\prime 2}-X_{j h}^{2}}}- \\
-\arcsin \frac{X_{j} Z_{k h}}{\sqrt{t^{\prime \prime 2}-Z_{k h}^{2}} \sqrt{t^{\prime 2}-X_{j}^{2}}}
\end{array}\right)+ \\
& +\frac{\left|Z_{k h}\right| t^{\prime \prime}}{Z_{k}}\left(\begin{array}{c}
\arcsin \frac{X_{j h} Z_{k}}{\sqrt{t^{\prime 2}-Z_{k}^{2}} \sqrt{t^{\prime 2}-X_{j h}^{2}}}- \\
-\arcsin \frac{X_{j} Z_{k}}{\sqrt{t^{\prime 2}-Z_{k}^{2}} \sqrt{t^{\prime 2}-X_{j}^{2}}}
\end{array}\right)
\end{aligned}
$$

When integrating Equation (14) over the third variable, we use the following equality for the first four terms with arcsin: 
$\int d t^{\prime \prime} \arcsin \frac{Z_{k h}}{\sqrt{t^{\prime 2}-X_{j h}^{2}}}=t " \arcsin \frac{Z_{k h}}{\sqrt{t^{\prime 2}-X_{j h}^{2}}}+$

$+Z_{k h} \ln \left|\frac{t "+\sqrt{t^{\prime \prime 2}-X_{j h}^{2}-Z_{k h}^{2}}}{\sqrt{X_{j h}{ }^{2}+Z_{k h}^{2}}}\right|+$

$+X_{j h} \frac{Z_{k h}}{\left|Z_{k h}\right|} \arcsin \frac{X_{j h} \sqrt{t^{\prime 2}-X_{j h}^{2}-Z_{k h}^{2}}}{Z_{k h} \sqrt{t^{\prime 2}-X_{j h}^{2}}}$,

where used integration by parts

$\int d t^{\prime \prime} \arcsin \frac{Z_{k h}}{\sqrt{t^{\prime 2}-X_{j h}^{2}}}=t^{\prime \prime} \arcsin \frac{Z_{k h}}{\sqrt{t^{\prime \prime 2}-X_{j h}^{2}}}+$

$+Z_{k h} \int d t^{\prime \prime} \frac{t^{\prime \prime 2}}{\left(t^{\prime 2}-X_{j h}{ }^{2}\right) \sqrt{t^{\prime 2}-X_{j h}{ }^{2}-Z_{k h}^{2}}}$

and formulae from [16]:

$\int \frac{d x}{\left(x^{2}-b^{2}\right) \sqrt{x^{2}-a^{2}}}=\frac{1}{b \sqrt{a^{2}-b^{2}}} \arcsin \frac{b \sqrt{x^{2}-a^{2}}}{a \sqrt{x^{2}-b^{2}}}$,

where $x>a^{2}>b^{2}$.

The last two terms in Equation (14) are integrated as follows. At first, we use integrating by parts:

$\int d t^{\prime \prime} t " \arcsin \frac{b c}{\sqrt{t^{\prime 2}-b^{2}} \sqrt{t^{\prime 2}-c^{2}}}=$

$=\frac{1}{2} \int d u \arcsin \frac{b c}{\sqrt{u^{2}-b^{2}} \sqrt{u^{2}-c^{2}}}=$

$=\frac{u}{2} \arcsin \frac{b c}{\sqrt{u^{2}-b^{2}} \sqrt{u^{2}-c^{2}}}+$

$+\frac{b c}{4} \int d u\left(\frac{1}{u-c^{2}}+\frac{1}{u-b^{2}}\right) \frac{u}{\sqrt{u^{2}-u\left(b^{2}+c^{2}\right)}}$.

And then, substitution of

$\sqrt{u\left(u-b^{2}-c^{2}\right)}=u v$,

where

$v=\sqrt{\left(u-b^{2}-c^{2}\right) / u}$,

$u=\left(b^{2}+c^{2}\right) /\left(1-v^{2}\right)$,

gives us:

$\int d u\left(\frac{1}{u-c^{2}}+\frac{1}{u-b^{2}}\right) \frac{u}{\sqrt{u^{2}-u\left(b^{2}+c^{2}\right)}}=$

$=2\left(\frac{c}{b} \arctan \frac{c v}{b}+\frac{b}{c} \arctan \frac{b v}{c}+\ln \left|\frac{1+v}{1-v}\right|\right)$

As a result, the final expression for Equation (18) will have the following form:

$$
\begin{aligned}
& \int d t^{\prime \prime} t " \arcsin \frac{b c}{\sqrt{t^{\prime 2}-b^{2}} \sqrt{t^{\prime \prime 2}-c^{2}}}= \\
& =\frac{t^{\prime \prime}}{2} \arcsin \frac{b c}{\sqrt{t^{\prime \prime 2}-b^{2}} \sqrt{t^{\prime \prime 2}-c^{2}}}+ \\
& +\frac{1}{2}\left[\begin{array}{l}
c^{2} \arctan \frac{c}{b} \sqrt{\frac{t^{\prime \prime 2}-b^{2}-c^{2}}{t^{\prime \prime 2}}}+ \\
+b^{2} \arctan \frac{b}{c} \sqrt{\frac{t^{\prime 2}-b^{2}-c^{2}}{t^{\prime \prime}}}+ \\
+2 \ln \frac{t^{\prime \prime}+\sqrt{t^{\prime \prime 2}-b^{2}-c^{2}}}{\sqrt{b^{2}+c^{2}}}+
\end{array}\right]
\end{aligned}
$$

For ease of use, let's split the final expression for triple integral from Equation (7) into parts according with Equation (14):

$$
\begin{aligned}
& \int_{T_{i}}^{T_{i h}} d t " \int_{X_{j}}^{X_{j h}} d x " \int_{Z_{k}}^{Z_{\text {hlh }}} d z^{\prime \prime} \frac{1}{\sqrt{t^{\prime 2}-x^{\prime 2}-z^{12}}}= \\
& =I_{1}-I_{2}+I_{3}-I_{4}-I_{5}+I_{6},
\end{aligned}
$$

where parts are:

$$
\begin{aligned}
& I_{1}=X_{j h} \int_{T_{i}}^{T_{i h}} d t^{\prime \prime}\left(\arcsin \frac{Z_{k h}}{\sqrt{t^{\prime 2}-X_{j h}^{2}}}-\arcsin \frac{Z_{k}}{\sqrt{t^{\prime 2}-X_{j h}^{2}}}\right) \\
& I_{2}=X_{j} \int_{T_{i}}^{T_{i h}} d t^{\prime \prime}\left(\arcsin \frac{Z_{k h}}{\sqrt{t^{\prime 2}-X_{j}^{2}}}-\arcsin \frac{Z_{k}}{\sqrt{t^{\prime 2}-X_{j}^{2}}}\right) \\
& I_{3}=Z_{k h} \int_{T_{i}}^{T_{i h}} d t "\left(\arcsin \frac{X_{j h}}{\sqrt{t^{\prime 2}-Z_{k h}^{2}}}-\arcsin \frac{X_{j}}{\sqrt{t^{\prime \prime 2}-Z_{k h}^{2}}}\right) \\
& I_{4}=Z_{k} \int_{T_{i}}^{T_{i h}} d t "\left(\arcsin \frac{X_{j h}}{\sqrt{t^{\prime 2}-Z_{k}^{2}}}-\arcsin \frac{X_{j}}{\sqrt{t^{\prime 2}-Z_{k}^{2}}}\right)
\end{aligned}
$$

$I_{5}=\frac{\left|Z_{k h}\right|}{Z_{k h}} \int_{T_{i}}^{T_{i h}} d t^{\prime \prime \prime} t\left(\begin{array}{l}\arcsin \frac{X_{j h} Z_{k h}}{\sqrt{t^{\prime 2}-Z_{k h}^{2}} \sqrt{t^{\prime 2}-X_{j h}^{2}}}- \\ -\arcsin \frac{X_{j} Z_{k h}}{\sqrt{t^{\prime 2}-Z_{k h}^{2}} \sqrt{t^{\prime 2}-X_{j}^{2}}}\end{array}\right)$

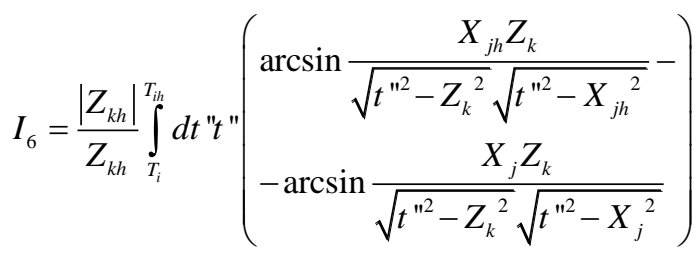

It is important to note that:

- $I_{2}$ can be obtained from $I_{1}$ by replacing $X_{j h} \rightarrow X_{j}$,

- $I_{3}$ can be obtained from $I_{1}$ by replacing $X_{j h} \rightarrow Z_{k h}, Z_{k h} \rightarrow X_{j h}, X_{j} \rightarrow Z_{k}, Z_{k} \rightarrow X_{j}$,

- $I_{4}$ can be obtained from $I_{3}$ by replacing $Z_{k h} \rightarrow Z_{k}$,

- $I_{6}$ can be obtained from $I_{5}$ by replacing $Z_{k h} \rightarrow Z_{k}$. 
Thus, it is only necessary to calculate explicitly the expressions for Equation (24) and (28).

The explicit form of $I_{1}$ and $I_{5}$ are as follows:

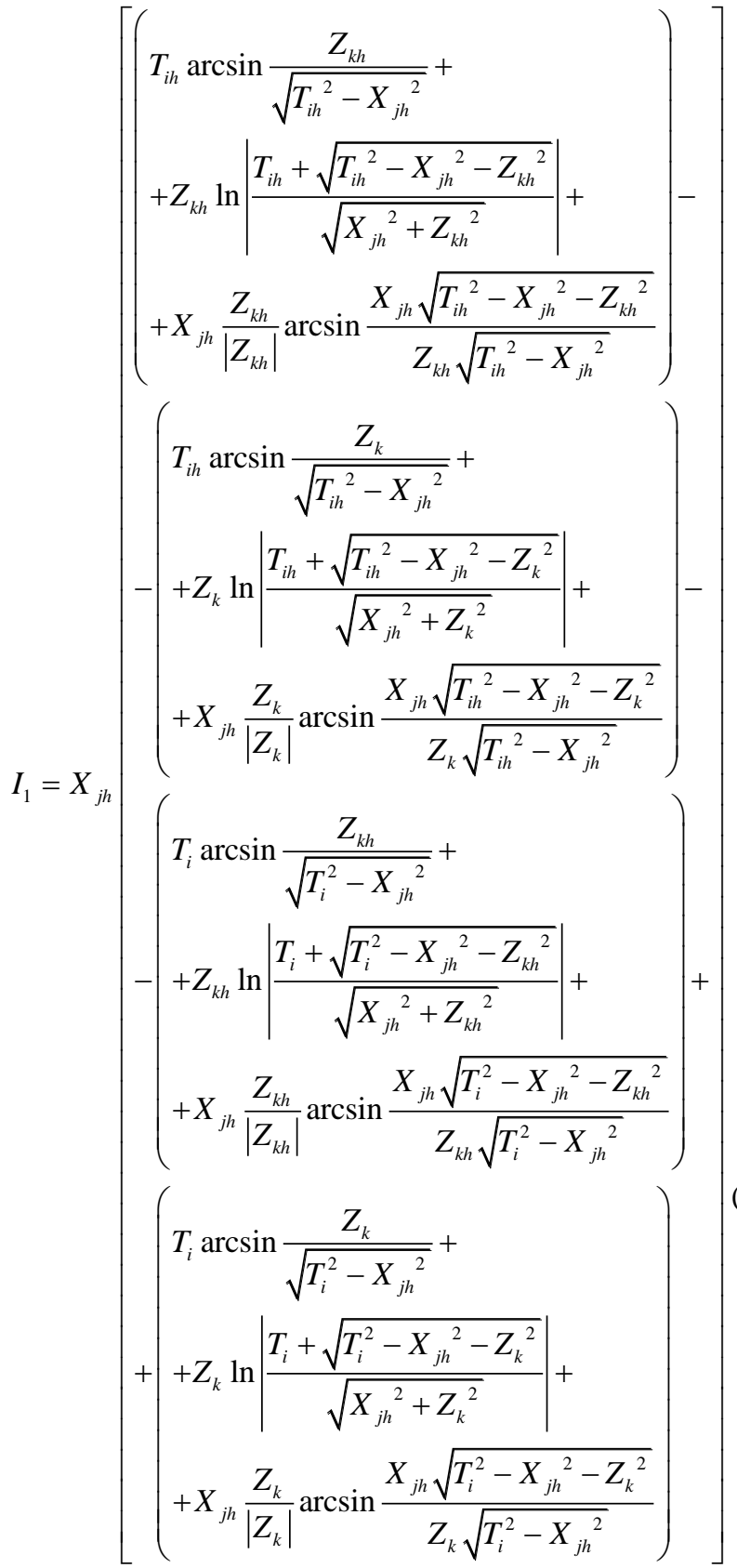

\section{CONCLUSION}

In the article presented the extension of the approximating functions method to solve electrodynamics problems in a planar waveguide with non-magnetic media with losses for the case of constant polarization inside it in threedimensional space and time domain using the Volterra integral equation method.

The explicit expression for calculating integral part of equation in one mesh cell is obtained. It contains only

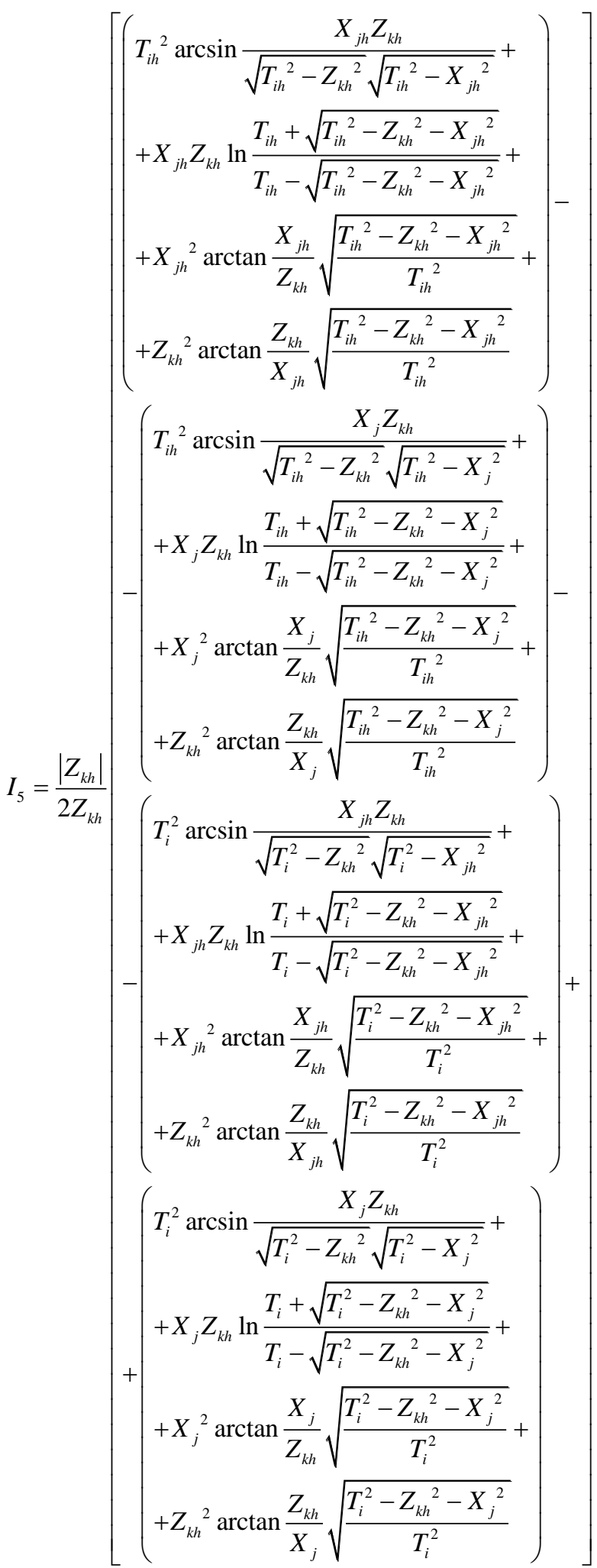

simple arithmetic operations, which are the fastest for calculations in computer software, square root, arcsine and natural logarithm, which have tables of exact values or approximations built into programming languages.

In addition, the final expression consists of 6 terms, which are interrelated, and only two of them need to be calculated to get expressions for all the others. 


\section{AUTHOR CONTRIBUTIONS}

Conceptualization, formal analysis, analytical transformations, validation, color graphics, writing original draft preparation, writing - review and editing, D. Zolotariov,

Methodology, formal analysis, analytical transformations, $\mathrm{b} / \mathrm{w}$ graphics, A. Nerukh.

\section{CONFLICTS OF INTEREST}

The authors declare that there is no conflict of interest regarding the publication of this article.

\section{REFERENCES}

1. Y. Shifman, Y. Leviatan, "On the use of spatiotemporal multiresolution analysis in method of moments solutions of transient electromagnetic scattering," IEEE Trans. on Antennas and Propagation, vol.49, pp. 1123, 2001.

2. M. R. Gomez, A. Salinas, A. R. Bretones, "Time-Domain Integral Equation Methods For Transient Analysis," IEEE Antennas and Propagation Magazine, vol. 34, pp. 15-24, 1992.

3. A. G. Nerukh, N. K. Sakhnenko, T. Benson, P. Sewell, Non-stationary electromagnetics (Singapore: Pan Stanford Publishing Ltd., 2013).

4. A. Nerukh, and T. Benson, Non-Stationary Electromagnetics: An Integral Equations Approach (2nd ed.) (Jenny Stanford Publishing, 2018), https://doi.org/10.1201/9780429027734.

5. J. N. Reddy, An Introduction to the Finite Element Method (Third ed. (McGraw-Hill, 2005).

6. J. Jin, The Finite Element Method in Electromagnetics (NY: Wiley 1993).

7. J. F. Lee, R. Lee, A. Cangellaris, "Time-Domain Finite-Element Methods," IEEE Trans. Antennas Propagat, vol. 45, pp. 430-442, 1997.

8. D. Anish, A. Dasgupta, G. Sarkar, "A new set of orthogonal functions and its application to the analysis of dynamic systems," Journal of the Franklin Institute, vol. 343, pp. 1-26, 2006.

9. K. Maleknejad, H. Almasieh, M. Roodaki, "Triangular functions (TF) method for the solution of nonlinear Volterra-Fredholm integral equations," Commun Nonlinear Sci Numer Simulat, vol. 10, pp. 10-12, 2010.

10. D. Zolotariov, A. Nerukh, "Extension of the approximation functions method for 2D nonlinear Volterra integral equations," Applied Radioelectronics, vol. 10, no. 1, pp. 39- 44, 2011.

11. A. Nerukh, D. Zolotariov and T. Benson, "The approximating functions method for nonlinear Volterra integral equations," Opt Quant
Electron, vol. 47, pp. 2565-2575, 2015, https://doi.org/10.1007/s11082-015-0141-2.

12. D. Zolotariov, "The New Modification Of The Approximating Functions Method For Cloud Computing," International Journal Of Mathematics And Computer Research, vol. 9, no. 9, pp. 2376-2380, 2021, https://doi.org/10.47191/ijmcr/v9i9.01.

13. D. Zolotariov, "The mechanism for creation of event-driven applications based on Wolfram Mathematica and Apache Kafka," Innovative Technologies and Scientific Solutions for Industries, vol. 15, no. 1, pp. 53-58, 2021, https://doi.org/10.30837/ITSSI.2021.15.053.

14. D. Zolotariov, "The platform for creation of event-driven applications based on Wolfram Mathematica and Apache Kafka," Innovative Technologies and Scientific Solutions for Industries, vol. 16, no. 2, pp. 12-18, 2021, https://doi.org/10.30837/ITSSI.2021.16.012.

15. D. Zolotariov, A. Nerukh, "The study of application of approximating functions method for problems in a planar waveguide with nonmagnetic media with losses," (publishing)

16. Yu.A. Brychkov, O. I. Marichev, A.P. Prudnikov, Tables of Indefinite Integrals: A Handbook (Russia: Fizmatlit, 2003) (In Russian). 